\title{
First Principles Calculation of $\varepsilon$-Phase of Solid Oxygen
}

\author{
K.H. Kamaruddin ${ }^{a}$, A.F.Z. Abedin ${ }^{a}$, N.A. Zabidi ${ }^{b * *}$, M.Z.A. YAhyA ${ }^{a}$, M.F.M. TAiB ${ }^{c}$ \\ AND A.N. ROSLI ${ }^{d}$ \\ ${ }^{a}$ Defence Science Department, Faculty of Defence Science and Technology, Universiti Pertahanan Nasional Malaysia, \\ Kuala Lumpur 57000, Malaysia \\ ${ }^{b}$ Physics Department, Centre for Defence Foundation Studies, Universiti Pertahanan Nasional Malaysia, \\ Kuala Lumpur 57000, Malaysia \\ ${ }^{c}$ Faculty of Applied Sciences, Universiti Teknologi MARA, 40450 Shah Alam, Selangor, Malaysia \\ ${ }^{d}$ Faculty of Science and Technology, Universiti Sains Islam Malaysia, Nilai 71800, Negeri Sembilan, Malaysia
}

The electronic structures of $\varepsilon$-phase of solid oxygen $\left(\mathrm{O}_{2}\right)_{4}$ are studied within the framework of densityfunctional theory. The intriguing molecule has been known to have magnetic properties at room temperature by applying pressure. Nevertheless, until now there was no evidence of band structure studied in the antiferromagnetic behaviour of $\left(\mathrm{O}_{2}\right)_{4}$. We report a comparison study for spin and non-spin polarization orbital which suggests that this ferromagnetic configuration of $\left(\mathrm{O}_{2}\right)_{4}$ could not be seen experimentally, and antiferromagnetic configuration of $\left(\mathrm{O}_{2}\right)_{4}$ was seen at higher pressure of about $10 \mathrm{GPa}$. The antiferromagnetic state transforms into the superconducting state as the sample temperature decreases. The results can serve as a useful approximation in studying general features of the electronic structure. The $\left(\mathrm{O}_{2}\right)_{4}$ clusters are reported in the Raman study, having significant absorption at $1516 \mathrm{~cm}^{-1}$ below infrared region.

DOI: 10.12693/APhysPolA.129.468

PACS/topics: 74.20.Pq, 74.70.-b, 31.15.E-, 75.50.Xx

\section{Introduction}

The diatomic molecule of oxygen with valency configuration of $2 s^{2} p^{4}[1]$ is one of interest which carries magnetic moment with a spin number $S=1$ [2]. At present, six phases of solid oxygen having distinct crystallographic properties at different pressure and temperature $(P-$ $T)[3,4]$ are established unambiguously. A seventh phase has been observed under high pressure but the magnetic interaction which is essential for the molecular arrangement is not well established [5]. As solid oxygen becomes denser, its volume practically decreases while its color becomes darker. Under very high pressure, solid oxygen which can be considered as "spin-controlled crystal" [2] changes its magnetic order [4] and becomes a metal and, at low temperature of $0.6 \mathrm{~K}$, it transforms to a superconducting state, assigned by neutron-diffraction study [6]. The epsilon phase of solid oxygen $\left(\varepsilon-\mathrm{O}_{2}\right)$ is red in color. It is an intriguing organic compound as compared to the other phases of solid oxygen and exhibits a stable liquid form of oxygen at pressure about $8 \mathrm{GPa}[7]$ at room temperature. The $\varepsilon-\mathrm{O}_{2}$ contains four $t_{2}$ molecules and thus forms rhomboid cluster $\left(\mathrm{O}_{2}\right)_{4}$ molecular units [8] which are chemically weakly bonded by the van der Waals forces between $\mathrm{O}_{2}$ molecules $[9,10]$. The crystallography of $\varepsilon$ $\mathrm{O}_{2}$ was experimentally confirmed to have $C 2 / m\left(C_{2 h}^{3}\right)$ symmetry assigned to the monoclinic structure by powder X-ray [9-11] and single-crystal diffraction [12]. Previous study suggested that $\varepsilon-\mathrm{O}_{2}$ has no long-range mag-

\footnotetext{
*corresponding author; e-mail: noriza@upnm.edu.my
}

netic order and shows peculiar behavior as magnetically collapsed [13], where the spin and non-spin polarized orbital calculations were found to have similar total energies [14]. In this paper, we report the electronic band structure and density of states obtained from the first principles electronic structure calculations as well as the vibrational frequency of $\varepsilon$ phase of solid oxygen.

\section{Materials and method}

\subsection{Materials}

All calculations were made based on experimental structures under high pressure $[9,10]$. The structure contains three $\left(C_{2 h}^{3}\right)$ symmetry inequivalent atoms; two (oxygen $\mathrm{O} 1$ and $\mathrm{O} 2$ ) occupy $4 \mathrm{i}$ positions of the monoclinic cell of $C 2 / m$ space group at $(x, 0, z)$ and the third $(\mathrm{O} 3)$ at $8 \mathrm{j}$ position at $(x, y, z)$. For ease, there are two $\mathrm{O}_{8}$ clusters in the monoclinic cell. One is placed at the origin of the lattice and the other is at the center of the $a b$ plane. The characterized structure is shown
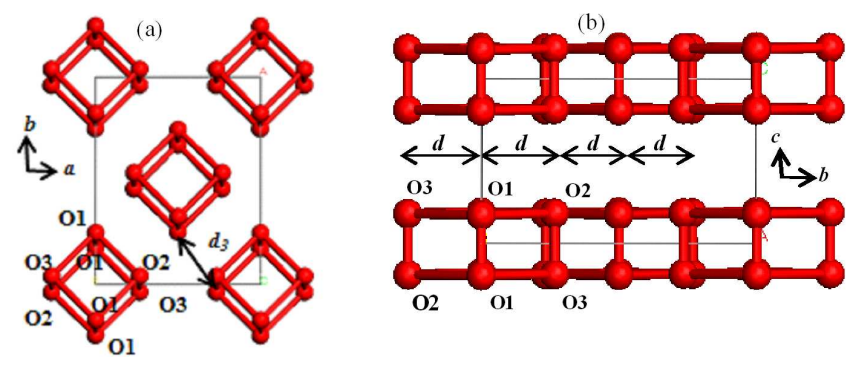

Fig. 1. The $\varepsilon-\mathrm{O}_{2}$ showing (a) a periodic form of $C 2 / m$ symmetry and (b) the intracluster, $d_{1}$ and $d_{2}$, as well as the intercluster distances, $d_{3}$. 
in Fig. 1a and $\mathrm{b}$ at different views and the lattice configurations with atomic coordinates are given in Table I. The electronic band structure obtained at $L, M, A, G, Z$, and $V$ of $k$-points was necessary to achieve convergence in the sampling of the Brillouin zone. After optimization at minimum energy, an isolated $\mathrm{O}_{8}$ cluster is found to have changed in the intracluster distances reported in Table II. The intercluster distances, $d_{3}$ are $2.661 \AA$ and $2.610 \AA$ for model 1 and model 2, respectively.

TABLE I

The structural parameters used in the DFT calculation.

\begin{tabular}{|c|c|c|c|}
\hline \multicolumn{2}{|c|}{ Pressure [GPa] } & Model 1 & Model 2 \\
\hline \multirow{4}{*}{$\begin{array}{c}\text { lattice } \\
\text { parameters }\end{array}$} & $a[\AA]$ & 8.141 & 7.77 \\
\hline & $b[\AA]$ & 5.747 & 5.501 \\
\hline & $c[\AA]$ & 3.773 & 3.657 \\
\hline & $\beta[\operatorname{deg}]$ & 117.07 & 116.4 \\
\hline \multirow{3}{*}{$\begin{array}{c}\text { atomic } \\
\text { coordinates }\end{array}$} & O1 & $(0.029,0.266,0.177)$ & $(0.826,0,0.175)$ \\
\hline & $\mathrm{O} 2$ & $(0.247,0,0.177)$ & $(0.247,0,0.192)$ \\
\hline & O3 & $(0.199,0,-0.177)$ & $(0.0379,0.2666,0.185)$ \\
\hline
\end{tabular}

TABLE II

The intracluster distances before and after optimization at minimum energy.

\begin{tabular}{c|c|c|c|c}
\hline \hline \multirow{2}{*}{ Distances } & \multicolumn{2}{|c|}{ Model 1 } & \multicolumn{2}{c}{ Model 2 } \\
\cline { 2 - 5 } & Before $[\AA]$ & After $[\AA]$ & Before $[\AA]$ & After $[\AA]$ \\
\hline O3-O1 $\left(d_{1}\right)$ & 2.342 & 2.074 & 2.180 & 2.077 \\
O1-O2 $\left(d_{2}\right)$ & 2.349 & 2.075 & 2.193 & 2.077 \\
O1-O1 & 1.198 & 1.207 & 1.203 & 1.207 \\
O2-O3 & 1.197 & 1.207 & 1.212 & 1.208
\end{tabular}

\subsection{Methods}

Self-consistent electronic structure calculations were performed within the framework of density-functional theory $[15,16]$ through the Materials Studio (accelrys) package which was based on the all-electron orbital calculations. For most of the calculations, the local density approximation (LDA) and local spin-density approximation in the Perdew and Wang scheme [17] were used to describe the exchange-correlation effect. The generalized gradient approximation (GGA) form of Perdew, Burke and Ernzerhof [18] has been used and the results were found similar to LDA as in the previous studies $[19,20]$. Hence, the reported values are only in the LDA. We optimized the structural and lattice parameters for the minimum energy. The vibrational frequency of the isolated cluster of $\left(\mathrm{O}_{2}\right)_{4}$ was obtained at the accuracy of convergence better than $0.01 \mathrm{eV}$. The total energies of the optimized structures were then determined using the $k$-point sampling grid along high symmetry directions in the Brillouin zone for primitive unit cell which contains a single unit cell in the lattice structure. The band structure and density of states (DOS) were obtained in the coordinates of $k$-points:
$L(-0.5,0,0.5), M(-0.5,-0.5,0.5), A(-0.5,0,0)$, $G(0,0,0), Z(0,-0.5,0.5)$, and $V(0,0,0.5)$.

\section{Results and discussion}

Figures 2, 3 and 4 show the band structure and density of states of $\left(\mathrm{O}_{2}\right)_{4}$ molecules in Ha unit. The calculations were performed using two different spin configurations. The non-spin polarization was obtained by forcing double occupancy of each band of the models at different pressures. Model 1 (Fig. 2b) shows the Fermi level to lie within the narrow $\mathrm{O} 2 p$ band at 1.02 states/per unit cell of density of states. By allowing unrestricted spin polarized within the unit cell, model 1 of $\left(\mathrm{O}_{2}\right)_{4}$ indicates ferromagnetic material where the bands are found slightly split at the ground state as shown in Fig. 3a and c. The ferromagnetic results of the minority spin state associated with $\mathrm{O}$ sites spin up separate from the minority spin down. The spin up are occupied more than the spin down eigenstates. The total energies calculated for spin and non-spin polarized models are found to be essentially the same and therefore the $\left(\mathrm{O}_{2}\right)_{4}$ has been claimed to be nonmagnetic. The phase transforms to nonmagnetic ordered as the sample temperature decreases indicating dissipation of magnetic order [13]. The localized magnetic moments vanished as $\left(\mathrm{O}_{2}\right)_{4}$ materials become superconducting.

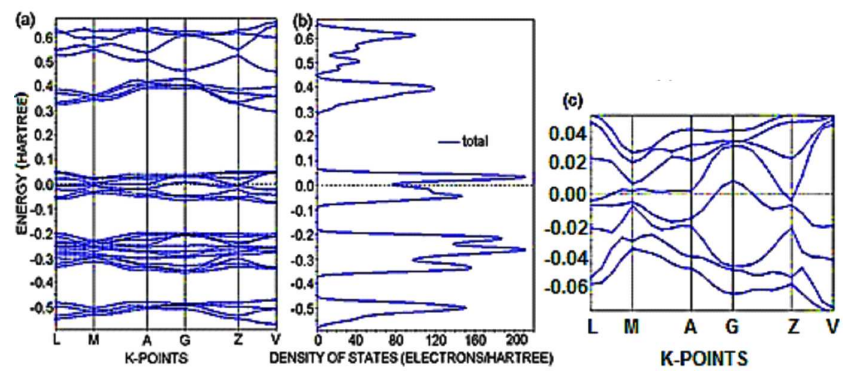

Fig. 2. The (a) band structure, (b) density of states (DOS) and (c) the enlargement of band structure at the Fermi energy, $E_{\mathrm{F}}$ are obtained using non-spin polarized calculation for model 1 at $11.4 \mathrm{GPa}$.
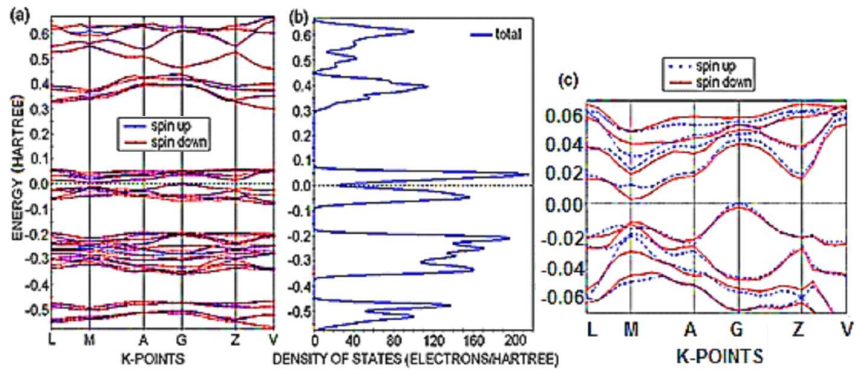

Fig. 3. The (a) band structure, (b) density of states (DOS) and (c) magnified band structure at the Fermi level $E_{\mathrm{F}}$, results using spin polarized calculation for model 1 .

At low pressure, $\left(\mathrm{O}_{2}\right)_{4}$ exhibits a change in behaviour where ferromagnetic materials turn into antiferromagnetic phase (Fig. 4a) as pressure increases, with zero total 

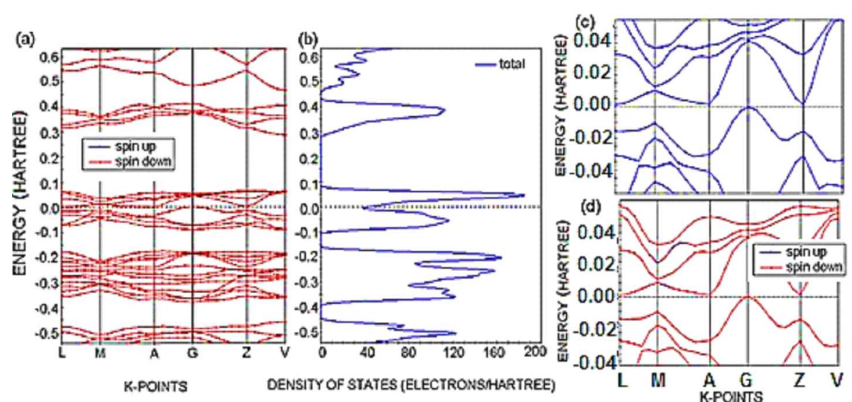

Fig. 4. The (a) band structure, and (b) density of states (DOS) of model 2 calculated by using spin polarized orbitals within LDA. The (c) magnified band structure of non-spin polarized, and (d) the magnified band structure of spin polarized calculation at Fermi level $E_{\mathrm{F}}$.

magnetization in the absence of external magnetic field. The result shows only the plot of spin up contributions, since spin down distribution is identical with the spin up. The antiferromagnetic is obtained by forcing opposite spins on sites related by inversion symmetry within the unit cell, consistent with experimental antiferromagnetic structure at low temperature. The indirect band gaps for model 1 as shown in Fig. 2c are $0.025 \mathrm{eV}$ at $M-$ $L, 0.185 \mathrm{eV}$ at $M-A$ and $0.446 \mathrm{eV}$ at $V-Z$. We find the minimum indirect gap energy of $G-A$ at $0.044 \mathrm{eV}$ for model 2 (Fig. 4c) to be approximately similar to the one established in theoretical study [21] which performed pseudopotential plane-wave method in the calculation. The direct gaps results for both models are given in Table III.

TABLE III

The direct energy gaps of models 1 and 2 at different $k$-points symmetry.

\begin{tabular}{|c|c|c|c|c|c|c|}
\hline & $L$ & $M$ & $A$ & $G$ & $Z$ & $V$ \\
\hline $\begin{array}{c}\text { Model } 1 \\
\text { energy }[\mathrm{eV}]\end{array}$ & 0.083 & 0.1902 & 0.4762 & 0.6164 & 0.083 & 1.7763 \\
\hline $\begin{array}{c}\text { Model } 2 \\
\text { energy }[\mathrm{eV}]\end{array}$ & 0.4735 & 0.5667 & 0.8716 & 1.1282 & 0.4735 & 2.4513 \\
\hline
\end{tabular}

Further, the observed Raman spectra in $\varepsilon-\mathrm{O}_{2}$ phase have fewer vibrational modes as shown in Fig. 5. Only $G$-point is used when performing electron-phonon dispersion in the Raman study. The vibrational modes were calculated for an $\varepsilon-\mathrm{O}_{2}$ phase which is found to reach significant absorption at $1512 \mathrm{~cm}^{-1}$ for model 1 (Fig. 5a) and $1516 \mathrm{~cm}^{-1}$ for model 2 (Fig. 5b) similarly to the Raman values in previous study [8]. The higher peak of model 1 (Fig. 5a) shows two frequencies having the same value which contributes to degeneracy, as compared to the model 2 (Fig. 5b) which shows two significant frequency and results in one highest peak which give useful information in determining various characteristics of their structure.
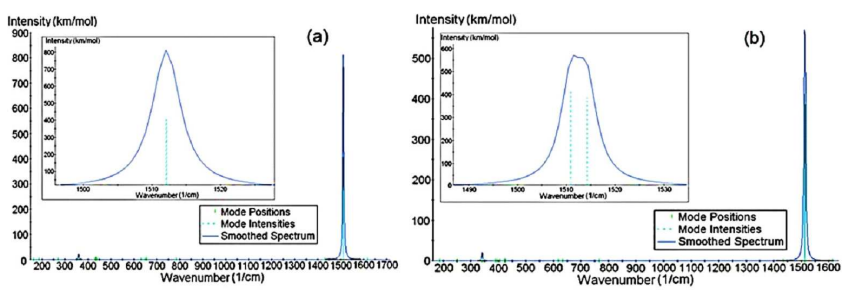

Fig. 5. The vibrational frequency of (a) model 1 (the higher peak shows two frequencies having the same value contributing to degeneracy, shown in inset), and (b) model 2 (the higher peak showing two different frequencies, shown in inset).

\section{Conclusion}

We have calculated the band gaps of $\varepsilon-\mathrm{O}_{2}$ phase for which $p$ band has the greatest electron density at the Fermi surface. At higher pressure, it is found that in the DOS the structure exhibits metallic behaviour under normal state and becomes superconducting under cooling at high temperature, while at lower pressure, $10 \mathrm{GPa}$, the structure exhibits ferromagnetic behaviour. There exists coupling between molecules causing the magnetic moments to switch from one molecule to the other. This results in the occurrence of antiferromagnetic material at higher pressure.

\section{Acknowledgments}

The authors would like to thank the Universiti Pertahanan Nasional Malaysia for the support and the project is supported by Ministry of Education under grant scheme (FRGS/1/2013/ST05/UPNM/02/2) for the high speed computer and the software package. We thank Mohd Sazwan Affendi for computational methods and suggestions.

\section{References}

[1] D. Vandebilt, Phys. Rev. B 41, 7892 (1990).

[2] Y.A. Freiman, H.J. Jodl, Phys. Rep. 401, 1 (2004).

[3] Y. Akahama, H. Kawamura, O. Shimomura, Phys. Rev. B 64, 054105 (2001).

[4] I.N. Goncharenko, O.L. Makarova, L. Ulivi, Phys. Rev. Lett. 93, 055502 (2004).

[5] T. Nomura, Y.H. Matsuda, S. Takeyama, A. Matsuo, K. Kindo, J.L. Her, T.C. Kobayashi, Phys. Rev. Lett. 112, 247201 (2014).

[6] R.J. Meier, R.B. Helmholdt, Phys. Rev. B 29, 1387 (1984).

[7] M. Nicol, K. Syassen, Phys. Rev. B 28, 1201 (1983).

[8] F.A. Gorelli, L. Ulivi, M. Santoro, R. Bini, Phys. Rev. Lett. 83, 4093 (1999).

[9] L.F. Lundegaard, G. Weck, M.I. McMahon, Nature 443, 201 (2006).

[10] H. Fujihisa, Y. Akahama, H. Kawamura, Y. Ohishi, O. Shimomura, H. Yamawaki, M. Sakashita, Y. Gotoh, S. Takeya, K. Honda, Phys. Rev. Lett. 97, 085503 (2006). 
[11] Y. Akahama, H. Kawamura, D. Häusermann, M. Hanfland, O. Shimomura, Phys. Rev. Lett. 74, 4690 (1995).

[12] G. Weck, P. Loubeyre, R. LeToullec, Phys. Rev. Lett. 88, 035504 (2002).

[13] I.N. Goncharenko, Phys. Rev. Lett. 94, 205701 (2005).

[14] J.B. Neaton, N.W. Ashcroft, Phys. Rev. Lett. 88, 205503 (2002).

[15] P. Hohenberg, W. Kohn, Phys. Rev. 136, B864 (1964).

[16] W. Kohm, L.J. Sham, Phys. Rev. 140, A1133 (1965).

[17] J.P. Perdew, Y. Wang, Phys. Rev. B 45, 13244 (1992).
[18] J.P. Perdew, K. Burke, M. Ernzerhof, Phys. Rev. Lett. 77, 3865 (1996).

[19] A.N. Rosli, N.A. Zabidi, H.A. Kassim, K.N. Shrivastava, J. Cluster Sci. 21, 197 (2010).

[20] N.A. Zabidi, H.A. Kassim, K.N. Shrivastava, AIP Conf. Proc. 1017, 326 (2008).

[21] Y.-H. Liu, Z.-M. Liu, Y.-M. Ma, Z. He, F.-B. Tian, T. Cui, B.-B. Liu, G.-T. Zou, Chin. Phys. Lett. 24, 11 (2007) 